\title{
Efficient Full-Wave Modeling of Radiative Near-Field Interactions in Semi-Anechoic Conditions
}

\author{
Gert-Jan Stockman ${ }^{1}$, Hendrik Rogier ${ }^{1}$ and Dries Vande Ginste ${ }^{1}$.
}

\begin{abstract}
In this paper, a full-wave method to efficiently compute the electromagnetic interaction between two devices placed in semi-anechoic conditions is proposed. The aim of this research is the accurate and efficient reproduction of radiated immunity and emission tests in simulation. The employed technique relies on a single simulation (or measurement) of the radiation pattern of each device and allows an arbitrary relative position between the devices. The resulting procedure is practical, has a low computational cost, and shows good agreement with reference solutions.
\end{abstract}

Index Terms-Semi-Anechoic, Electromagnetic Interference (EMI), Radiated Immunity and Emission.

\section{INTRODUCTION}

D ESIGN of electronic applications requires profound inspection of the Electromagnetic Compatibility (EMC) behavior of the system, not only to obtain reliable and robust design, but also to pass legislative and regulatory requirements. Looking at Electromagnetic Interference (EMI), both radiated emission and susceptibility aspects require careful examination. Compliance tests for the assessment of radiated emission or immunity are often carried out in an anechoic chamber. The Device Under Test (DUT) is then rotated and a new measurement is performed for every angular position. Semi-anechoic chambers are used as well, especially for measurements at lower frequencies (low $\mathrm{kHz}$ or even low $\mathrm{Hz}$ range). Standards for this low range do not specify a need for absorption or anechoic behavior since the current absorber technology cannot deliver absorption for these frequencies [1]. Furthermore semi-anechoic chambers are much more practical when performing tests on large objects such as cars.

The goal is to take radiated emission and immunity into account during the design phase (or precompliance phase). In the ideal case, this is done via simulations, although often not a trivial task. A single simulation requires large computational resources in order to achieve accurate results.

To relax these high computational requirements, many methods have been developed to mimic (aspects of) the large electromagnetic problem. In [2] and [3] different emission models are described. When looking at susceptibility, various hybrid techniques have been proposed. In [4], model-reduction techniques are combined with full-wave solvers. In [5], a hybrid method, based on the Finite-Difference Time-Domain

\footnotetext{
${ }^{1}$ IBCN/Electromagnetics Group, Department of Information Technology, Ghent University/iMinds, Sint-Pietersnieuwstraat 41, B-9000 Gent, Belgium. E-mail: gertjan.stockman@UGent.be, Tel.: +3292643593.
}

(FDTD) algorithm and a field coupling model of transmission lines are employed. Furthermore, [6] and [7] discuss susceptibility of transmission line networks with linear/nonlinear load terminations. Another way to reduce computational resources is by so-called Domain Decomposition Methods (DDMs). Nonconformal Finite Element (FE)-based DDMs have been reported in [8] and [9]. Furthermore, interactions have also been described via a hybrid Physical Optics (PO)/generalizedscattering-matrix approach [10]. Recently, in [11], a DDM-like approach was suggested to model interactions by using eigenmodal expansions. In [12], multilayer high-speed interconnects are modelled using modal ports.

In this paper, we will not consider emission or susceptibility separately, but the electromagnetic interaction between radiating devices. The novelty of our method lies in the fact that it is based purely on a single simulation (or measurement) of the radiation patterns of each individual device. Besides reducing the computational burden of modeling an entire set-up, another advantage is that the devices may be moved without requiring new simulations (or measurements). As such, the technique is very tractable and opens the way to the efficient reproduction of radiated emission and immunity tests in simulations.

Work related to this topic has already been performed in [13]. The modeling of the interaction between two single-port radiating devices (in the radiative near-field, also known as the Fresnel region) that have arbitrary relative positions and orientations in free space (anechoic conditions) was described. The difference w.r.t. this work is that we expand the formalism to account for semi-anechoic conditions, maintaining the efficiency and accuracy of the advocated method.

In Section II, the electromagnetic interaction between devices in semi-anechoic conditions is described and the formalism to efficiently calculate them is explained. Section III contains a validation example to prove the correctness of our technique, whereas in Section IV some application examples are considered, showing its applicability. The conclusion to this work together with an outline for future research is given in Section V.

In the sequel, all sources and fields are assumed to be time harmonic with angular frequency $\omega$ and time dependencies $e^{j \omega t}$ are suppressed. Unit vectors are denoted with a "hat", e.g. $\hat{\mathbf{v}}$.

\section{FORMALISM}

The general problem geometry for the case of a transmitting and receiving device placed in semi-anechoic conditions is 
shown in Fig. 1. The devices are represented by their current density sources $\mathbf{j}_{\mathrm{TX}}\left(\mathbf{r}^{\prime}\right)$ for the transmitter and $\mathbf{j}_{\mathrm{RX}}\left(\mathbf{r}^{\prime}\right)$ for the receiver. Due to the presence of the perfect electrically conducting (PEC) floor, an image source mirrored around the PEC floor (Fig. 2) is presented by means of the current density $\mathbf{j}_{\mathrm{TX}}^{m}\left(\mathbf{r}^{\prime \prime}\right)$. Throughout the rest of this paper, the superscript $m$ will be used to denote the mirrored fictitious device. The transmitter, mirrored transmitter and receiver are defined in volumes $V_{\mathrm{TX}}, V_{\mathrm{TX}}^{m}$ and $V_{\mathrm{RX}}$, respectively and appropriate phase centers $\mathcal{O}_{\mathrm{TX}}, \mathcal{O}_{\mathrm{TX}}^{m}$ and $\mathcal{O}_{\mathrm{RX}}$ are attached to them. The electric

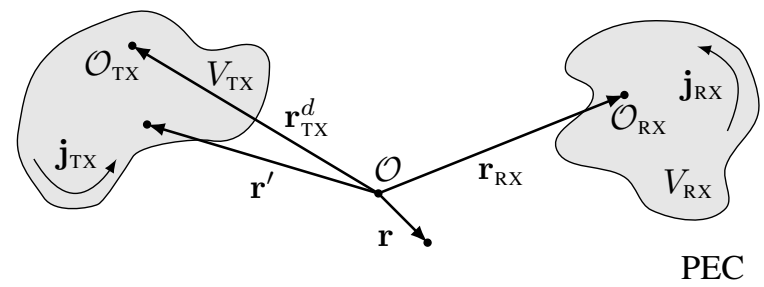

Fig. 1: General configuration of interacting devices in a semianechoic chamber.

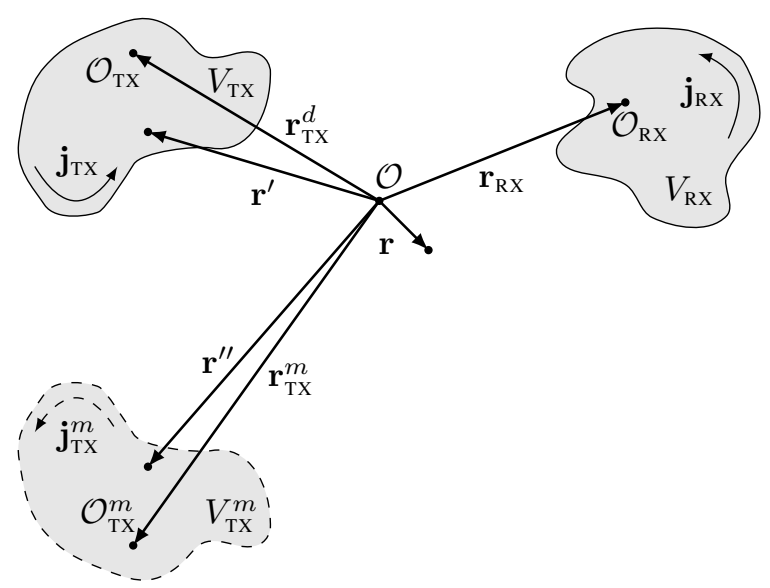

Fig. 2: Equivalent configuration of the interacting devices in a semi-anechoic chamber (Fig. 1). The PEC floor is replaced by a mirrored image source.

field generated by the transmitter is given by the Electric Field Integral Equation (EFIE) [14]:

$$
\mathbf{e}(\mathbf{r})=-j \omega \mu \int_{V} \mathcal{G}_{\text {semi }}\left(\mathbf{r}, \mathbf{r}^{\prime}\right) \cdot \mathbf{j}\left(\mathbf{r}^{\prime}\right) \mathrm{d} \mathbf{r}^{\prime},
$$

where $\mathbf{j}\left(\mathbf{r}^{\prime}\right)$ represents the current densities on all of the devices $\left(\mathbf{j}_{\mathrm{TX}}\left(\mathbf{r}^{\prime}\right), \mathbf{j}_{\mathrm{TX}}^{m}\left(\mathbf{r}^{\prime \prime}\right)\right.$ and $\left.\mathbf{j}_{\mathrm{RX}}\left(\mathbf{r}^{\prime}\right)\right)$. $\mu$ is the permeability of the background media, and $\mathcal{G}_{\text {semi }}\left(\mathbf{r}, \mathbf{r}^{\prime}\right)$ the three-dimensional dyadic Green's function in the semi-anechoic case, which equals

$$
\begin{aligned}
\mathcal{G}_{\text {semi } i}\left(\mathbf{r}, \mathbf{r}^{\prime}\right) & =\mathcal{G}_{\text {anechoic }}\left(\mathbf{r}, \mathbf{r}^{\prime}\right)+\mathcal{G}_{\text {mirror }}\left(\mathbf{r}, \mathbf{r}^{\prime}\right) \\
& =\left[\mathcal{I}+\frac{1}{k^{2}} \nabla \nabla\right]\left(\frac{e^{-j k\left|\mathbf{r}-\mathbf{r}^{\prime}\right|}}{4 \pi\left|\mathbf{r}-\mathbf{r}^{\prime}\right|}-\frac{e^{-j k\left|\mathbf{r}-\mathbf{r}^{\prime \prime}\right|}}{4 \pi\left|\mathbf{r}-\mathbf{r}^{\prime \prime}\right|}\right),
\end{aligned}
$$

with $\mathbf{r}^{\prime \prime}=\mathbf{r}^{\prime}-2 z^{\prime} \hat{\mathbf{z}}$ and $z^{\prime}=\mathbf{r}^{\prime} \cdot \hat{\mathbf{z}}$ when the origin of the coordinate system is chosen to reside on the PEC plane.
The integration domain $V$ in (1) extends over all devices, i.e. $V=V_{\mathrm{TX}} \cup V_{\mathrm{TX}}^{m} \cup V_{\mathrm{RX}}$. In this contribution, we assume that the devices are spaced sufficiently far from each other, such that there is no coupling via the reactive near-field. For such devices, positioned in each other's Fresnel or Fraunhofer region, the field incident on the receiving device can be accurately approximated by restricting $V$ to $V_{\mathrm{TX}} \cup V_{\mathrm{TX}}^{m}$ in (1). By applying Gegenbauer's addition theorem [15] to the scalar 3-D Green's function and employing a plane wave expansion [16], the incoming electric field is written in terms of incoming and outgoing plane waves:

$$
\begin{aligned}
\mathbf{e}^{\mathrm{inc}}(\mathbf{r})= & -\frac{\omega \mu k}{(4 \pi)^{2}} \iint_{\Omega} e^{-j \mathbf{k} \cdot\left(\mathbf{r}-\mathbf{r}_{\mathrm{RX}}\right)}[\mathcal{I}-\hat{\mathbf{k}} \hat{\mathbf{k}}] \\
& \cdot\left[T\left(\mathbf{r}_{\mathrm{TX}, \mathrm{RX}}^{d}, \hat{\mathbf{k}}\right) \int_{V_{\mathrm{TX}}} e^{j \mathbf{k} \cdot\left(\mathbf{r}^{\prime}-\mathbf{r}_{\mathrm{TX}}\right)} \mathbf{j}_{\mathrm{TX}}\left(\mathbf{r}^{\prime}\right) \mathrm{d} \mathbf{r}^{\prime}\right. \\
& \left.+T\left(\mathbf{r}_{\mathrm{TX}, \mathrm{RX}}^{m}, \hat{\mathbf{k}}\right) \int_{V_{\mathrm{TX}}^{m}} e^{j \mathbf{k} \cdot\left(\mathbf{r}^{\prime \prime}-\mathbf{r}_{\mathrm{TX}}^{m}\right)} \mathbf{j}_{\mathrm{TX}}^{m}\left(\mathbf{r}^{\prime \prime}\right) \mathrm{d} \mathbf{r}^{\prime \prime}\right] \mathrm{d} \hat{\mathbf{k}},
\end{aligned}
$$

where we defined $\mathbf{r}_{\mathrm{TX}, \mathrm{RX}}^{i}=\mathbf{r}_{\mathrm{RX}}-\mathbf{r}_{\mathrm{TX}}^{i}$ with $i=\{m, d\}$. The superscript $d$ refers to the direct transmitter, whereas superscript $m$ refers to its image mirrored w.r.t. the PEC plane. According to image theory, the current density of the mirrored device $\mathbf{j}_{\mathrm{TX}}^{m}\left(\mathbf{r}^{\prime \prime}\right)$ is linked to that of the direct transmitter $\mathbf{j}_{\mathrm{TX}}\left(\mathbf{r}^{\prime}\right)$ as

$$
\mathbf{j}_{\mathrm{TX}}^{m}\left(\mathbf{r}^{\prime \prime}\right)=-\mathbf{j}_{\mathrm{TX}, t}\left(\mathbf{r}^{\prime}\right)+j_{\mathrm{TX}, z}\left(\mathbf{r}^{\prime}\right) \hat{\mathbf{z}},
$$

where the subscript $t$ stands for the components tangential to the PEC plane. Furthermore, in (4), we integrate over the Ewald sphere $\Omega$ and $\mathbf{k}$ is the wave vector in spherical coordinates:

$$
\mathbf{k}=k \hat{\mathbf{k}}=k(\sin \theta \cos \phi \hat{\mathbf{x}}+\sin \theta \sin \phi \hat{\mathbf{y}}+\cos \theta \hat{\mathbf{z}}),
$$

with $k=\omega \sqrt{\epsilon \mu}$ the wavenumber of the background medium and $\epsilon$ its permittivity. Also, $\mathcal{I}$ is the unit dyadic and $T\left(\mathbf{r}_{\mathrm{TX}, \mathrm{RX}}^{i}, \hat{\mathbf{k}}\right)$ the translation operator defined as

$$
T\left(\mathbf{r}_{\mathrm{TX}, \mathrm{RX}}^{i}, \hat{\mathbf{k}}\right)=\sum_{l=0}^{\infty}(2 l+1) j^{-l} h_{l}^{(2)}\left(k\left|\mathbf{r}_{\mathrm{TX}, \mathrm{RX}}^{i}\right|\right) P_{l}\left(\hat{\mathbf{k}} \cdot \hat{\mathbf{r}}_{\mathrm{TX}, \mathrm{RX}}^{i}\right),
$$

where $h_{l}^{(2)}(\cdot)$ the $l$-th order spherical Hankel function of the second kind and $P_{l}(\cdot)$ the Legendre polynomial of degree $l$. The radiation pattern of the (mirrored) transmitting device is defined as

$$
\mathbf{F}_{\mathrm{TX}}^{i}(\hat{\mathbf{k}})=\frac{j \omega \mu}{4 \pi} \hat{\mathbf{k}} \times\left[\hat{\mathbf{k}} \times \int_{V_{\mathrm{TX}}^{i}} e^{j \mathbf{k} \cdot\left(\mathbf{r}^{\prime}-\mathbf{r}_{\mathrm{TX}}^{i}\right)} \mathbf{j}_{\mathrm{TX}}^{i}\left(\mathbf{r}^{\prime}\right) \mathrm{d} \mathbf{r}^{\prime}\right],
$$

and the radiation pattern of the receiver can be written in a similar way.

Using these expressions, the incident electric field (4) is written in terms of the radiation patterns of the devices and a simplified expression for the short-circuit current induced on 
the receiver is obtained [17]:

$$
I_{s c}=-\frac{1}{Z V_{0}} \sum_{i=\{d, m\}} \iint_{\Omega} T\left(\mathbf{r}_{\mathrm{TX}, \mathrm{RX}}^{i}, \hat{\mathbf{k}}\right) \mathbf{F}_{\mathrm{TX}}^{i}(\hat{\mathbf{k}}) \cdot \mathbf{F}_{\mathrm{RX}}(-\hat{\mathbf{k}}) \mathrm{d} \hat{\mathbf{k}}
$$

where $Z=\sqrt{\frac{\mu}{\epsilon}}$ is the wave impedance of the background medium and $V_{0}$ a pertinent normalization factor, which evolves from the reciprocity theorem and depends on the normalized radiation pattern when antenna $i$ is operating in transmit mode. As the antennas' radiation patterns scale with $V_{0}$, this parameter can be chosen to be $1 \mathrm{~V}$.

In practice, (9) can only be approximated since the infinite sum that occurs in the translation operator (7) has to be truncated to a finite number of multipoles $L$. [15] explains how to select this number and the accuracy it entails.

It is interesting to note that in order to calculate the influence of transmitting (noisy) devices on a receiving (susceptible) device, in semi-anechoic conditions, only the measured or simulated radiation patterns of the two devices are needed. The radiation pattern of the transmitter $\mathbf{F}_{\mathrm{TX}}^{d}(\hat{\mathbf{k}})$ and the fictitious transmitter $\mathbf{F}_{\mathrm{TX}}^{m}(\hat{\mathbf{k}})$ are linked to each other since the radiation pattern simply needs to be mirrored with respect to the PEC plane. Furthermore, the devices may be positioned anywhere in space, as long as there is no coupling via the reactive nearfield. This leads to very efficient calculations in semi-anechoic conditions, in comparison to full-wave solutions, especially when one or both of the devices are repeatedly repositioned in space, i.e. when $\mathbf{r}_{\mathrm{TX}, \mathrm{RX}}^{i}$ is varied. The expression for the short-circuit current (9) can be modified to account only for purely anehoic conditions, by simply omitting the term that corresponds to the contribution of the mirrored device.

\section{VALIDATION EXAMPLE}

In order to validate the explained method, a numerical experiment is conducted where transmitter and receiver are both half-wavelength dipole antennas. The antennas are placed parallel to each other at a height of $h=1 \mathrm{~m}$ above an infinite PEC plane and separated by $d=3 \mathrm{~m}$ as shown in Fig. 3 . The

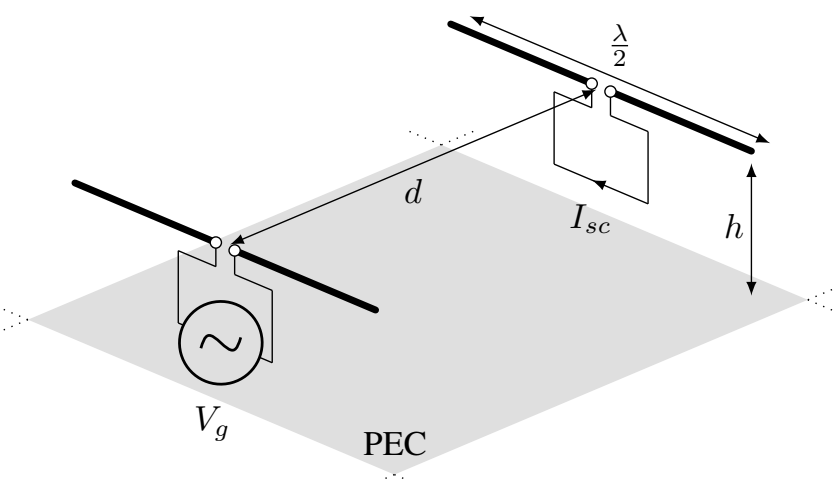

Fig. 3: Two parallel half-wavelength dipole antennas (separated by a distance $d=3 \mathrm{~m}$ ) are placed in an semi-anechoic chamber with a height of $h=1 \mathrm{~m}$ above the infinite PEC floor.

short-circuit current $I_{s c}$ calculated with our full-wave method
(9) is compared to a reference solution obtained by a Method of Moments (MoM) for arbitrary thin wires [18], but adapted to also account for the PEC plate. The thickness of the wires equals $10^{-4} \lambda$ and five segments are used to model a halfwavelength dipole. The transmitter is excited with $V_{g}=1 \mathrm{~V}$ between its terminals. The short-circuit current is found by inverting the full MoM matrix equation. The frequency (and thus also the physical length of the antenna) is swept and a comparison of the short-circuit current between the fullwave method and the MoM is made in Fig. 4. The radiation patterns used in the full-wave method are calculated from a straightforward MoM simulation for a single dipole. The number of multipoles used is $L=5$. For low frequencies, and

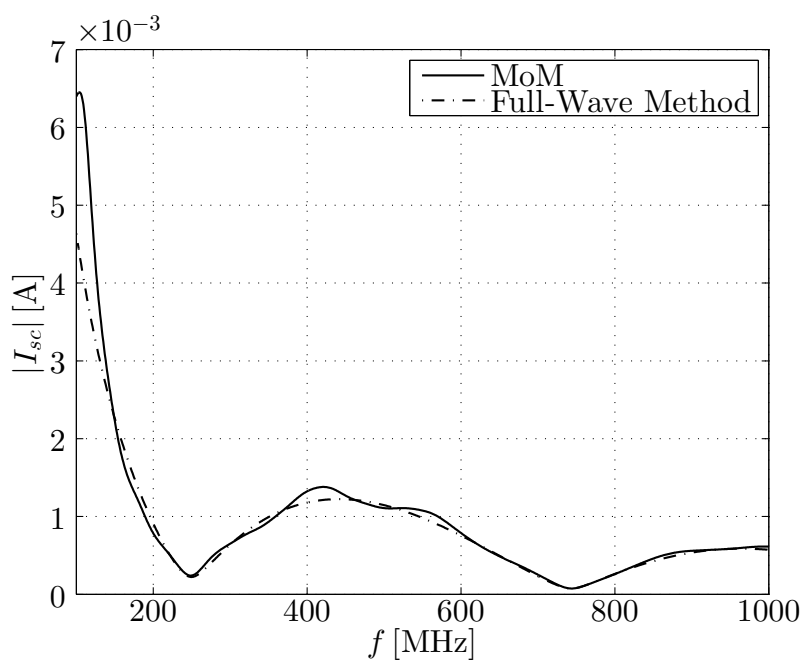

Fig. 4: Comparison of the amplitude of the short-circuit current at the receiving dipole between the full-wave method and the traditional MoM.

thus long wavelengths, the error starts to grow. The reason is that the electrical size of the antennas grows relative to their separation distance, which remains fixed to $3 \mathrm{~m}$. The result is that the devices come in proximity of each other's reactive near field and the devices become tightly coupled. The same reasoning should be used for the transmitter and its mirrored version, who also become coupled. Traditionally, devices are considered to be in each other's radiative nearfield, for distances between them not smaller than, say, $\lambda / 6$ [19]. Then, they may be treated as being uncoupled and our formalism is valid. For coupled devices, the approximation made on the integration domain in (1) is no longer valid and a multiport formalism is necessary. This is currently under investigation.

\section{ApPliCATION EXAMPLE}

\section{A. Shielding Efficiency}

A small thin-wire electric dipole antenna with a length of $4 \mathrm{~cm}$ is put inside a PEC shielding enclosure. The enclosure is a $50 \mathrm{~cm}$ side cubic box with a horizontal aperture in the front plane having dimensions $20 \mathrm{~cm} \times 5 \mathrm{~cm}$, as depicted in Fig. 5. The dipole antenna inside the enclosure is excited and another identical dipole antenna, placed $3 \mathrm{~m}$ in front of the 


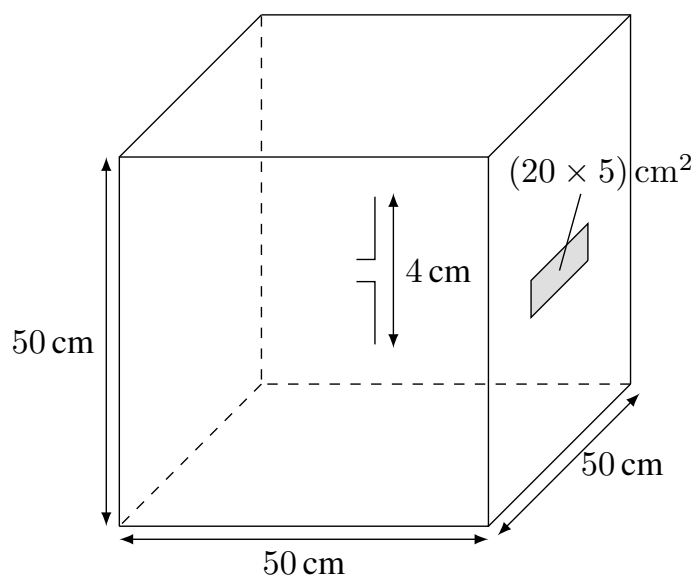

Fig. 5: Small thin-wire electrical dipole antenna inside a PEC metallic shielding enclosure. The enclosure is a $50 \mathrm{~cm}$ side cubic box with a horizontal aperture in the front plane.

slot, acts as a receiver. The bottom of the box is placed at a height of $1 \mathrm{~m}$ above a PEC floor. As a first step, Computer Simulation Technology Microwave Studio (CST MWS) is used to calculate the radiation patterns of the dipole antenna with and without the shielding enclosure (and without PEC plate). Subsequently, these radiation patterns are used as input for our full-wave method, allowing to efficiently calculate the short-circuit currents induced in the receiving dipole using (9). The short-circuit currents induced in the receiving antenna, are calculated with and without the shielding enclosure at the transmitter, leading to the currents $I_{s c, 1}$ and $I_{s c, 2}$ respectively. The Shielding Efficiency (SE) is defined as the ratio of the amplitude of these currents:

$$
\mathrm{SE}[\mathrm{dB}]=20 \log \left(\frac{\left|I_{s c, 2}\right|}{\left|I_{s c, 1}\right|}\right) \text {. }
$$

The SE calculated over a broad frequency range is shown in Fig. 6. Besides a computation where the PEC plate is considered, the anechoic case, i.e. the box is put in free space, where the term that accounts for the mirrored device is ommited in (9), is also calculated and shown. For the highest frequency, i.e. $1 \mathrm{GHz}$, the order of expansion is $L=18$. For the anechoic case, the obtained SE yields excellent agreement with the results shown in Fig. 5 of [20], proving the correctness of our method once again. Below $200 \mathrm{MHz}$, some small artifacts are visible, which are due to the radiation pattern simulations with CST MWS, suffering from inaccuracies as the bounding box is too small compared to the wavelength. Positioning the absorbing boundary conditions further away from the enclosure leads to unacceptably long simulaton times or even insufficient memory. This is also the reason why a complete set-up, including the receiving dipole antenna, cannot be simulated using CST MWS.

In the semi-anechoic case, the SE results are different, but the resonance peaks are not influenced. This is expected as these peaks only depend on the dimensions of the cavity. To better show the difference between the anechoic and semianechoic conditions, the result is shown for a limited frequency range in Fig. 7. Differences of up to $5 \mathrm{~dB}$ are observed,

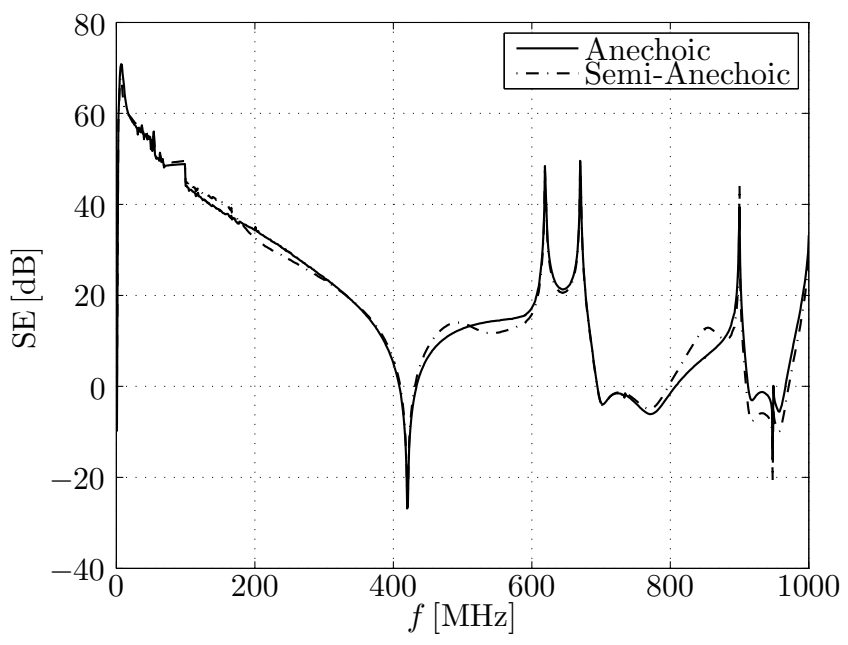

Fig. 6: SE as a function of frequency for a $50 \mathrm{~cm}$ side cubic box with a $20 \mathrm{~cm} \times 5 \mathrm{~cm}$ horizontal aperture centered in the front plane.

showing the importance of being able to properly account for the PEC plane during EMC testing.

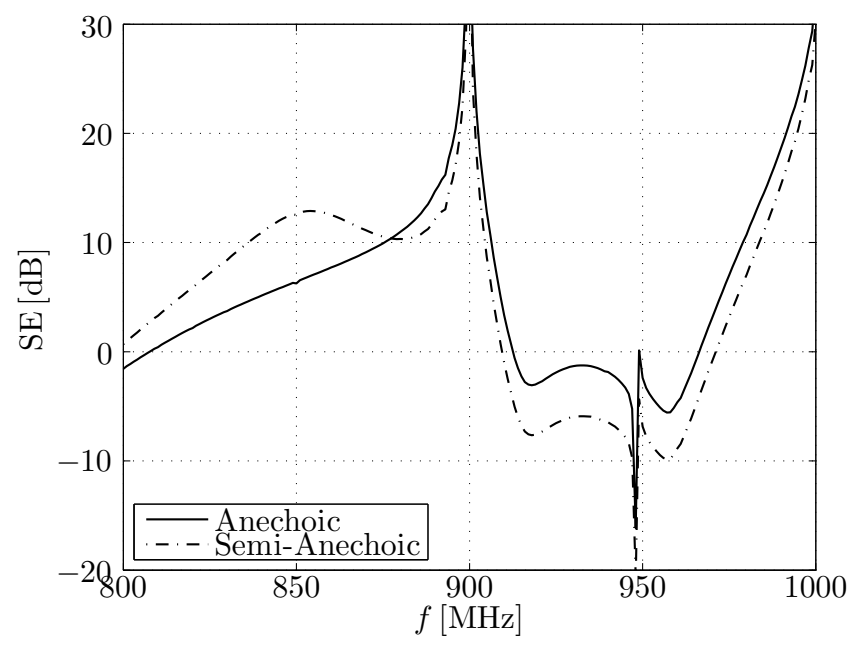

Fig. 7: Limited frequency range of the result shown in Fig. 6.

\section{B. Fading in a Semi-Anechoic Chamber}

In a second example, we apply our theory to two identical inset-fed microstrip patch antennas, designed to resonate at $1.5 \mathrm{GHz}$. The antennas are placed $h=1.75 \mathrm{~m}$ above the PEC floor. The setup configuration is shown in Fig. 8. The distance $d$ between the two antennas is varied within the range $[5 \lambda, 10 \lambda]$, and the influence of the reflection on the PEC floor is observed. To apply our method, the order of expansion is chosen to be $L=10$. The radiation patterns of the antennas are extracted from the 3-D planar full-wave solver Momentum from Advanced Design System (ADS) of Keysight Technologies, since they were designed using this software package. The result is shown in Fig. 9. As expected, due 


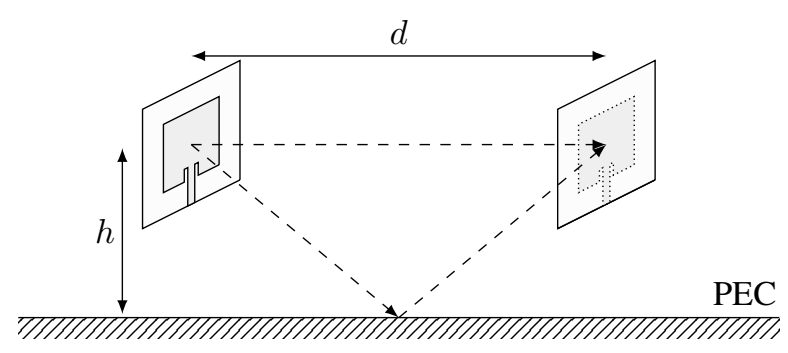

Fig. 8: Setup of two inset-fed microstrip patch antennas in a semi-anechoic chamber.

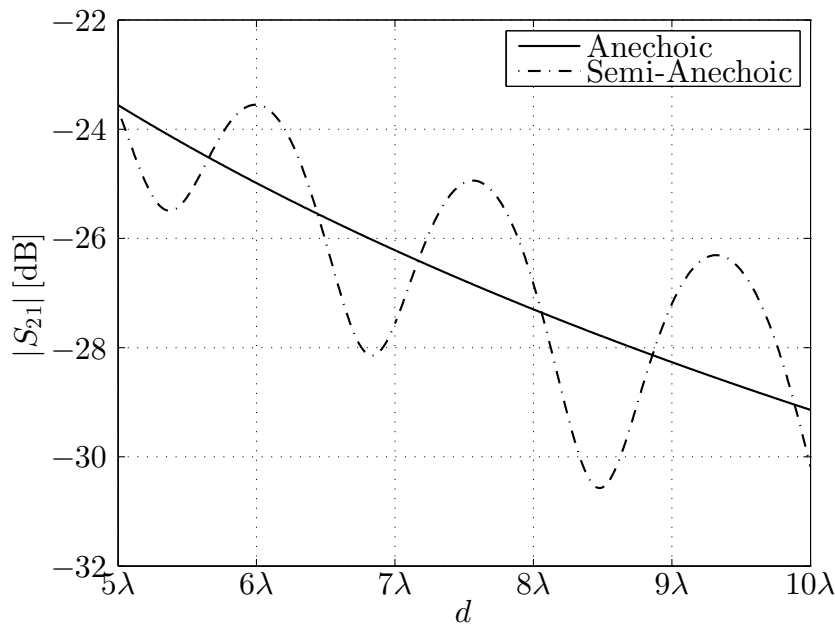

Fig. 9: Comparison of the configuration shown in Fig. 8 with and without PEC plate.

to constructive and destructive interference of the multipath present in this scenario, fading is observed in the link between the two devices.

\section{CONCLUSIONS}

The method developed in this paper allows for the efficient and accurate reproduction of immunity and emission tests in semi-anechoic conditions. The method relies on a single simulation or measurement of the radiation patterns of the devices, as such avoiding to have to model an entire (electrically large) set-up. Another advantage this method has over traditional simulations or measurements is that a repositioning of the devices does not entail a completely new computation or measurement. The correctness of the method was shown using a numerical validation. Practical examples were given to show the method's applicability.

Right now, the approach is only accurate as long as the coupling between the devices remains small. In other words, the devices should not reside in each others reactive near field. As they approach this zone, the coupling cannot be neglected anymore and a multiport approach to the problem is necessary, which is currently under investigation.

\section{REFERENCES}

[1] M. Wiles and V. Rodriguez, "Choosing the right chamber for your test requirements," EMC Directory \& Design Guide, May 2010.
[2] P. Li and L. J. Jiang, "Source reconstruction method-based radiated emission characterization for PCBs," IEEE Transactions on Electromagnetic Compatibility, vol. 55, no. 5, pp. 933-940, October 2013.

[3] P. Kralicek, W. John, R. De Smedt, K. Vervoort, and H. Garbe, "A voltage controlled emission model of electromagnetic emission of IC for system analysis," in IEEE International Symposium on Electromagnetic Compatibility, Montreal, Quebec, 13-17 August 2001, pp. 1197-1202.

[4] I. Erdin, M. Nakhla, and R. Achar, "Circuit analysis of electromagnetic radiation and field coupling effects for networks with embedded fullwave modules," IEEE Transactions on Electromagnetic Compatibility, vol. 42, no. 4, pp. 449-460, November 2000.

[5] M. Tang, J. Lu, J. Mao, and L. Jiang, "A systematic electromagneticcircuit method for EMI analysis of coupled interconnects on dispersive dielectrics," IEEE Transactions on Microwave Theory and Techniques, vol. 61, no. 1, pp. 1-13, January 2013.

[6] Y. Bayram and J. Volakis, "Hybrid S-parameters for transmission line networks with linear/nonlinear load terminations subject to arbitrary excitations," IEEE Transactions on Microwave Theory and Techniques, vol. 55, no. 5, pp. 941-950, May 2007.

[7] Z. Khan, Y. Bayram, and J. Volakis, "EMI/EMC analysis of printed circuit boards subject to near-zone illuminations," IEEE Transactions on Electromagnetic Compatibility, vol. 51, no. 2, pp. 406-408, May 2009.

[8] Y. Shao, Z. Peng, and J.-F. Lee, "Signal integrity analysis of high-speed interconnects by using nonconformal domain decomposition method," IEEE Transactions on Components, Packaging and Manufacturing Technology, vol. 2, no. 1, pp. 122-130, January 2012.

[9] Z. Peng, K.-H. Lim, and J.-F. Lee, "Nonconformal domain decomposition methods for solving large multiscale electromagnetic scattering problems," Proceedings of the IEEE, vol. 101, no. 2, pp. 298-319, February 2013.

[10] C. Della Giovampaola, E. Martini, A. Toccafondi, and S. Maci, "A hybrid $\mathrm{PO} /$ generalized-scattering-matrix approach for estimating the reflector induced mismatch," IEEE Transactions on Antennas and Propagation, vol. 60, no. 9, pp. 4316-4325, September 2012.

[11] T. Flisgen, H. Glock, and U. van Rienen, "Compact time-domain models of complex RF structures based on the real eigenmodes of segments," IEEE Transactions on Microwave Theory and Techniques, vol. 61, no. 6, pp. 2282-2294, June 2013.

[12] H. Wu and A. Cangellaris, "A finite-element domain-decomposition methodology for electromagnetic modeling of multilayer high-speed interconnects," IEEE Transactions on Advanced Packaging, vol. 31, no. 2, pp. 339-350, May 2008.

[13] G.-J. Stockman, H. Rogier, and D. Vande Ginste, "Efficient modeling of interactions between radiating devices with arbitrary relative positions and orientations," IEEE Transactions on Electromagnetic Compatibility, vol. 56, no. 6, pp. 1313-1321, December 2014.

[14] J. Van Bladel, Electromagnetic Fields. John Wiley \& Sons, 2007.

[15] W. C. Chew, J.-M. Jin, E. Michielssen, and J. Song, Fast and efficient algorithms in computational electromagnetics. Artech House Publishers, 2001.

[16] J. Stratton, Electromagnetic Theory. McGraw-Hill, 1941.

[17] J. Van Bladel, "On the equivalent circuit of a receiving antenna," IEEE Antennas and Propagation Magazine, vol. 44, no. 1, pp. 164-165, February 2002.

[18] W. C. Gibson, The Method of Moments in Electromagnetics. Chapman \& Hall/CRC, 2008, ch. 4, pp. 73-79.

[19] C. Balanis, Antenna Theory: Analysis and Design. John Wiley \& Sons, 2005.

[20] W. Wallyn, D. De Zutter, and H. Rogier, "Prediction of the shielding and resonant behavior of multisection enclosures based on magnetic current modeling," IEEE Transactions on Electromagnetic Compatibility, vol. 44, no. 1, pp. 130-138, Feb 2002. 